\title{
Real-time calibration of tied-arrays in the presence of atmospheric de-coherence
}

\author{
P.A. Fridman
}

\begin{abstract}
ASTRON, Oudehoogeveensedijk 4, Dwingeloo, 7991PD, The Netherlands
\end{abstract}
\begin{abstract}
Large radio astronomy multi-element interferometers are frequently used as single dishes in a tied-array mode when signals from separate antennas are added. Phase shifts arising during wave propagation through a turbulent atmosphere can significantly reduce the effective area of an equivalent single dish. Estimates of the impact of the ionosphere and troposphere on the effectiveness of a radio interferometer working in tied-array mode are given. A simple method of tied-array calibration using optimization techniques is proposed. Computer simulations demonstrate the efficacy of the proposed calibration algorithm.
\end{abstract}

\section{Introduction}

Large radio astronomy multi-element interferometers (VLA, WSRT) are frequently used in the tied-array mode where signals from separate antennas are added (Thompson et al. 2001, ch. 9.9). The output sum signal can be used in VLBI, pulsar and transients observations, SETI signals detection and the directto-Earth (DtE) reception of signals from cosmic apparatus. In all these cases a radio interferometer works as a single-dish antenna with one output. Partial signals from antennas are properly phased to collect emission from a point-like radio source in the sky and track it during its siderial movement. Standard calibration procedure using a correlator is employed to provide the necessary phase corrections for each individual antenna. Random phase perturbations such as phase shifts arising during wave propagation through the turbulent atmosphere can occur in the course of such observations. These phase errors reduce the total effective area of the tied-array and must be compensated for in real time.

New large scale projects such as SKA and LOFAR will also be operating in tied-array mode. The impact of ionospheric and tropospheric phase errors on the tied-array is calculated in this paper. A simple method of correcting these errors using the output signal of the tied-array is also proposed here.

\section{Tied-array with random phase errors}

The loss produced by the phase errors $\delta_{n, a t m}$ at the output of $n_{a^{-}}$ element array is equal to:

$$
L_{\delta, a t m}=\frac{1}{n_{a}^{2}} \sum_{n=1}^{n_{a}} \sum_{m=1}^{n_{a}} \exp \left[-\frac{D_{\delta, a t m}\left(b_{m n}\right)}{2}\right] .
$$

where $D_{\delta, a t m}\left(b_{m n}\right)=<\left(\delta_{n, a t m}-\delta_{m, a t m}\right)^{2}>$ is the variance of the random phase difference for the baseline $b_{m n}$. In the case of turbulent atmosphere $D_{\delta, a t m}\left(b_{m n}\right)$ is the structure phase function. The power-law (Kolmogorov) model will be used in the following sections to describe $D_{\delta, a t m}\left(b_{m n}\right)$ both for the ionosphere and troposphere phase fluctuations (Tatarskii 1978).

\subsection{Ionosphere}

The electron density $N$ in the ionosphere, considered as a func tion of spatial coordinates, has variations which are charac terized by a structure function $D_{N}(b)$ of electron density $N$ (Thompson et al. 2001, ch. 13). The ionosphere phase struc ture function is:

$$
D_{\text {ion }}(b)=2.91 r_{e}^{2} \lambda^{2} C_{N}^{2} h b^{5 / 3},
$$

where $b$ is the baseline, $C_{n}^{2}=\frac{r_{e}^{2} \lambda^{4}}{4 \pi^{2}} C_{N}^{2}, r_{e}=2.82 \cdot 10^{-15} \mathrm{~m}$ (electron radius), $\lambda$ is the wavelength, $h$ is the total propagation length through the irregularities of the ionosphere, $\lambda, h$ and $b$ must be substituted in meters. The value $C_{N}^{2}$ can be estimated by assuming that the ionosphere irregularities of electron density $\Delta(N)$ have a maximum dimension equal to $L_{0}$ :

$$
\Delta(N)^{2}=C_{N}^{2} L_{0}^{2 / 3}
$$

For example, for $\Delta(N) / N=0.01$ and $N=10^{12} \mathrm{~m}^{-3}$ (day time) we have for $L_{0}=10 \mathrm{~km}, C_{N}^{2}=2.154 \cdot 10^{17} \mathrm{~m}^{-20 / 3}$, for $\Delta(N) / N=0.03$ and $L_{0}=30 \mathrm{~km}, C_{N}^{2}=9.322 \cdot 10^{17} \mathrm{~m}^{-20 / 3}$. Figure 1a shows the square root of the ionosphere phase structure function for $N=10^{12} \mathrm{~m}^{-3}, \Delta(N) / N=0.01$ and $L_{0}=10 \mathrm{~km}, h=300 \mathrm{~km}$ calculated for three frequencies: $50 \mathrm{MHz}, 100 \mathrm{MHz}$ and $200 \mathrm{MHz}$.

Phase fluctuations can be also characterized by the Fried length:

$$
r_{\text {Fried }}=3.18 d_{0},
$$

where $d_{0}$ is the baseline at which $(2 \pi / \lambda) \sqrt{D_{\text {ion }}}=1 \mathrm{rad}$. For the parameters used in Fig. $1 \mathrm{a} r_{\text {Fried }}=1.16 \mathrm{~km}$ for $50 \mathrm{MHz}, r_{\text {Fried }}=2.66 \mathrm{~km}$ for $100 \mathrm{MHz}$ and $r_{\text {Fried }}=6.11 \mathrm{~km}$ for $200 \mathrm{MHz}$. Fig. 1b shows how Fried length depends on the frequency for the different values of $N, L_{0}, h$.

The loss caused by ionosphere random phase errors can be calculated in the example of the array whose configuration is shown in Fig. 3a . It is the random planar 100-element array with the coordinates $x_{i}$ and $y_{i}$ represented by random normal values with zero mean and standard deviation $S C$. For the array shown in Fig. 3a, $S C=1000 \mathrm{~m}$, therefore the maximum baseline is $\approx 5000 \mathrm{~m}$. Fig. $3 \mathrm{~b}$ demonstrates the dependance of loss versus array size $5 \cdot S C$. The curves are calculated for three frequencies: 50, 100 and $200 \mathrm{MHz}$. 


\subsection{Troposphere}

The troposphere phase structure function is (Stotskii 1973):

$$
\begin{aligned}
D_{\text {trop }}(b) & =2.91 k^{2} C_{l}^{2} b^{5 / 3}, L_{0}<b<L_{1} \\
& =2.91 k^{2} C_{L}^{2} b^{2 / 3}, L_{1}<b<L_{2} \\
& =2.91 k^{2} C_{L}, L_{2}<b,
\end{aligned}
$$

where $L_{0}$ and $L_{1}$ are the internal and external scales, respectively, of the isotropic three-dimensional turbulence model, $L_{0}=0.1-1 \mathrm{~cm}, L_{1}=5.6 \mathrm{~km}$ and $L_{2}=2000-3000 \mathrm{~km}$, the latter is determined by global meteorological variations. Factors $C_{l}^{2}$ and $C_{L}^{2}$ depend on the local content of water vapor and oxygen in the troposphere (weather conditions) and the values chosen for the purpose of calculation are $C_{l}^{2}=6.23 \cdot 10^{-11} \mathrm{~m}^{1 / 3}$ and $C_{L}^{2}=3.64 \cdot 10^{-7} \mathrm{~m}^{4 / 3}$.

Fig. 2a represents the structure function of electrical length (5) and Fig. 2b shows the Fried length as a function of the baseline. Fig. $3 c$ demonstrates the dependance of loss versus array size $5 \cdot S C$. The curves are calculated for three frequencies: 1400, 5000 and $8400 \mathrm{MHz}$.

\section{Simulation of real-time calibration}

Here a calibration method is proposed which uses points in the direct images of the field-of-view (FoV) with calibration sources. It is presumed that a full calibration with the correlator has already been performed before tied-array observation. The value of the signal power in the prescribed direction $\mathbf{u}_{s}$ in the image with one or several calibration sources available in FoV can be obtained by convolution of the sky intensity $B_{0}(\mathbf{u})$ with the instantaneous synthesized beam $P_{\text {inst }}\left(\mathbf{u}_{s}\right)$

$$
B_{\text {inst }}\left(\mathbf{u}_{s}\right)=B_{0}(\mathbf{u}) \oplus P_{\text {inst }}\left(\mathbf{u}_{s}\right),
$$

where $\oplus$ denotes convolution.

To eliminate $\delta_{n, \text { atm }}$, compensation phase shifts $\delta_{n, \text { comp }}$ are introduced at each $n$-th array element. Having the output of the total power detector $B_{\text {inst }}\left(\mathbf{u}_{s}\right)$ for the direction $\mathbf{u}_{s}$ we can maximize this value by varying phase shifts $\delta_{n, c o m p}$, i.e., we have an optimization problem for $n_{a}$ values of $\delta_{n, c o m p}$. In general, for each direction $\mathbf{u}_{s}$ this problem can be written as

$$
\widehat{\delta}_{n, \text { comp }}\left(\mathbf{u}_{s}\right)=\arg \max _{\delta_{n, \text { comp }}}\left[B_{\text {comp }}\left(\mathbf{u}_{s}\right)\right], n=1 \ldots n_{a}
$$

This scheme is illustrated in the following example of computer simulation. The 60 -element spatially random planar array is represented in Fig. 4a. The phase errors introduced in each of the array element signals are modeled by the two-dimensional random value, Fig. 4b, with circular symmetrical spatial spectral density which decreases radially according to the (-11/3) power law. The instantaneous sample of phase errors at frequency $100 \mathrm{MHz}$ as a function of baseline length is given in Fig. 4c.

The image containing three point sources is represented in Fig. $5 \mathrm{a}$ and the synthesized image in the presence of the phase errors (Fig. 4c) is shown in Fig. 5b (isoplanicity being presumed).

The value of the synthesized image in the direction of the largest source (lower left in the image) was used as the cost function. The genetic algorithm was applied because of the strong multi-modality of the cost function (7) and this algorithm finds the global maximum successfully.

After applying the optimization procedure and introducing the resulting compensation phases, the corrected image is shown in Fig. 5c (right panel).

The contour presentations in Fig. 6 correspond to the undistorted image (left panel), the image with phase errors (middle panel) and the image after correction (right panel), respectively.

\section{Conclusions}

1. The effective area of tied arrays may be significantly re duced by ionospheric and tropospheric phase irregularities at low and high frequencies, respectively.

2. Observations are made at times (VLBI, transients monitoring, DtE) when it is impossible to choose quiet atmospheric conditions and real-time calibration is necessary and has to be fulfilled in parallel with observations.

3. The total power at the auxiliary outputs of the tied-array phased in the direction of calibration sources, can be used on a level with traditional calibration methods using corre lators. Multi-beam facilities are necessary for creating these auxiliary outputs. Optimization algorithms (genetic algorithms, simulated annealing) can be used to compensate for propagation phase errors by maximizing the amplitude of a chosen calibration source. The tied array can preserve its correctly phased state during lengthy observations using one or several auxiliary outputs, thus working in the self-cohering regime.

\section{References}

Stotski, A. A. 1973, Radiophys. Quant. Electron, 16, 620

Tatarskii, V. I. 1978, Wave Propagation in Turbulent Media (John Wiley \& Sons, Inc.)

Thompson, A. R., Moran, J. M. \& Swenson, G. W. 2001 Interferometry and Synthesis in Radio Astronomy (John Wiley \& Sons, Inc.), ch. 9.9, ch.13 

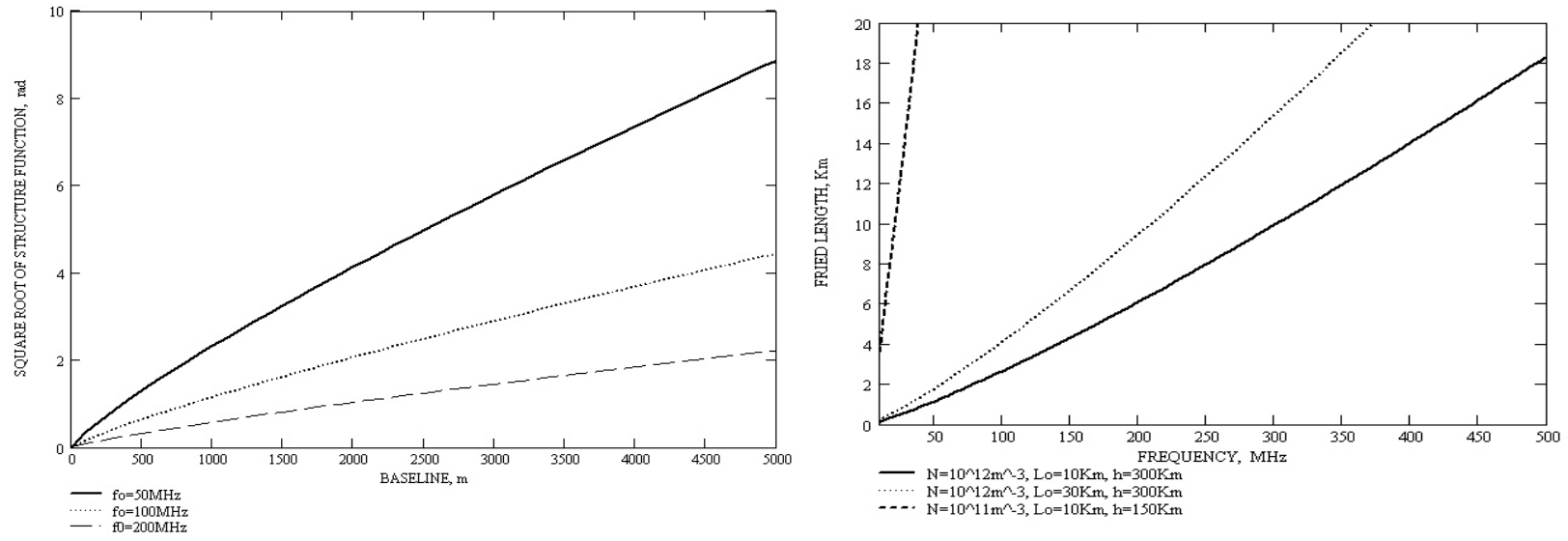

Fig. 1: a) left panel: square root of the ionosphere phase structure function of electrical length, $N=10^{12} \mathrm{~m}^{-3}, \Delta(N) / N=0.01$ and $L_{0}=10 \mathrm{~km} \Omega$ calculated for three frequencies: $50 \mathrm{MHz}, 100 \mathrm{MHz}$ and $200 \mathrm{MHz}$; b) right panel: Fried length for different values of $N, L_{0}, h$.
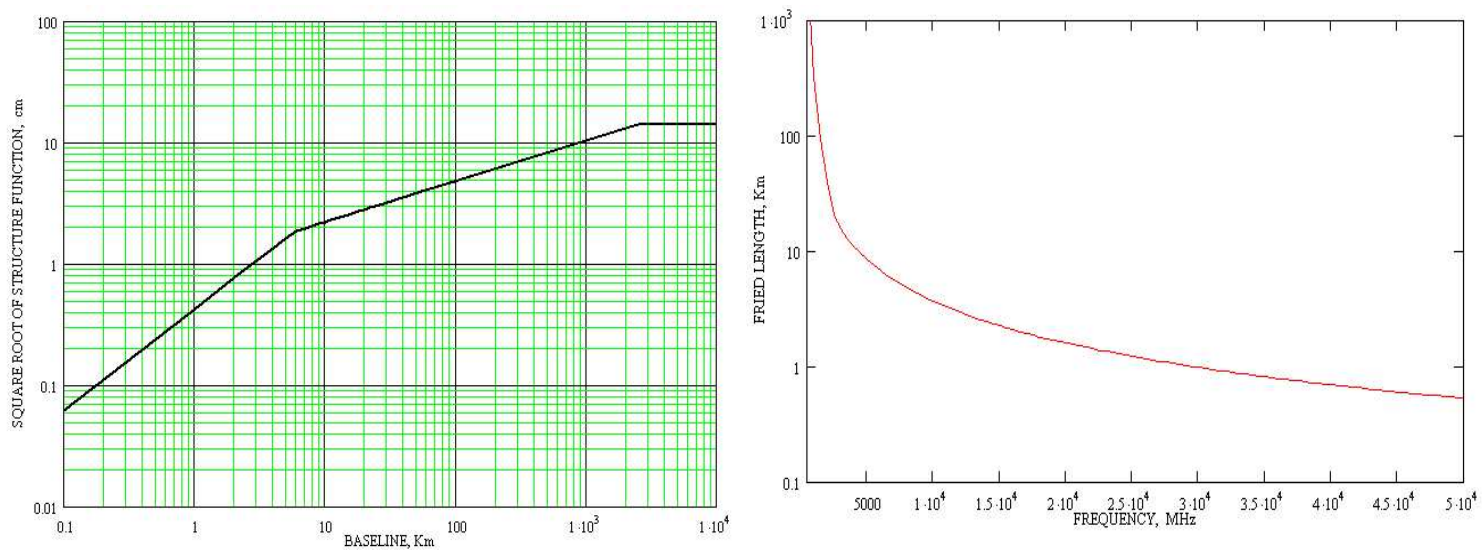

Fig. 2: a) left panel: Square root of the troposphere structure function of electrical length, in $\mathrm{cm}$; b) right panel: Fried length as a function of frequency.
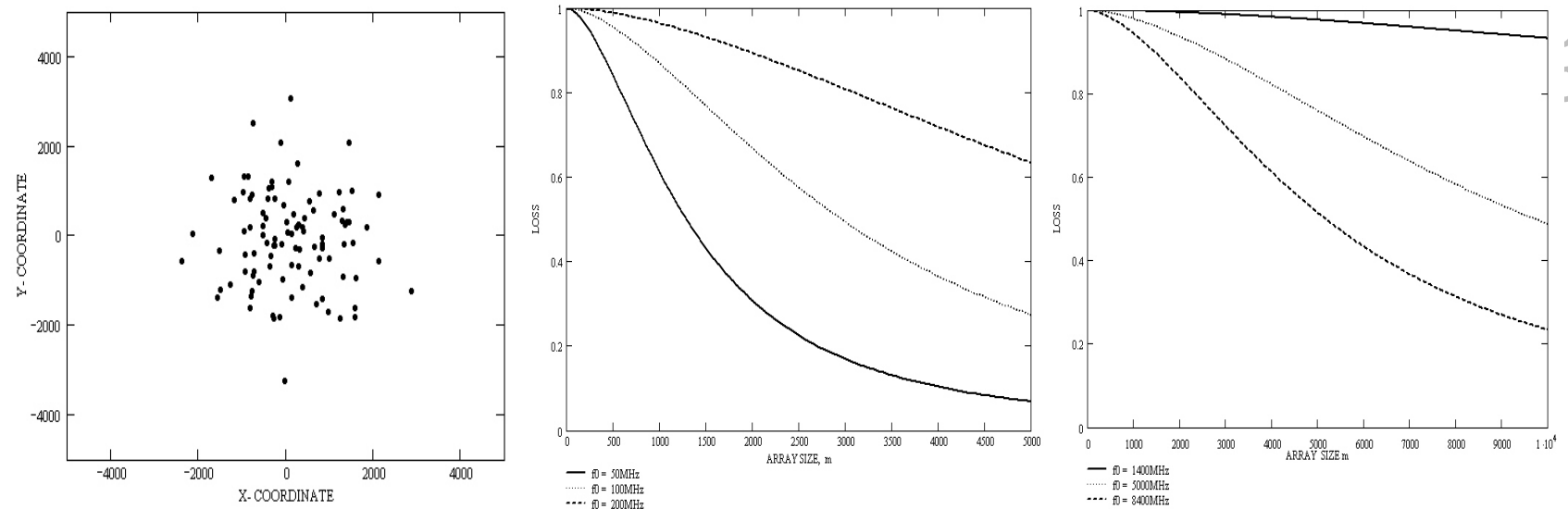

Fig. 3: a) left panel: random array configuration; b) middle panel: loss produced by phase fluctuations in the ionosphere calculated for three frequencies: 50, 100 and $200 \mathrm{MHz}$. The structure function from Fig. 1a is used; right panel: loss produced by phase fluctuations in the troposphere calculated for three frequencies: 1400, 5000 and $8400 \mathrm{MHz}$, the structure function from Fig. 2a is used. 

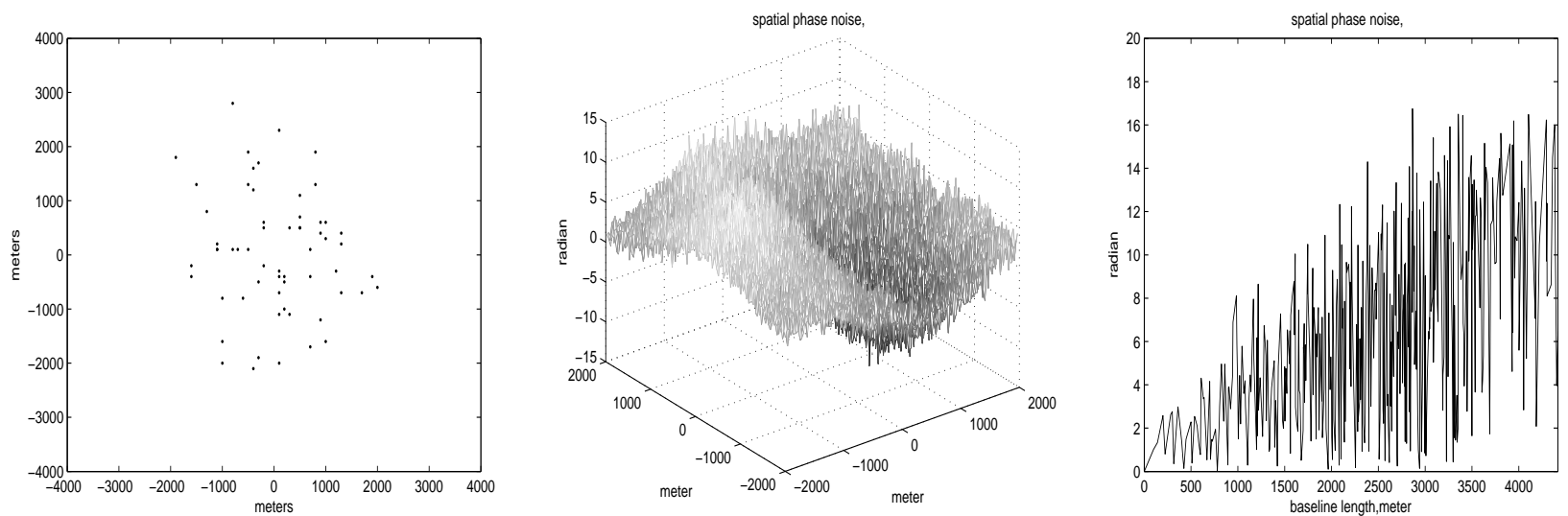

Fig. 4: a) left panel: 60-element tied array configuration; b) middle panel: spatial phase error distribution, projected on the array plane; c) right $\Omega$ panel: phase errors as function of baseline length.
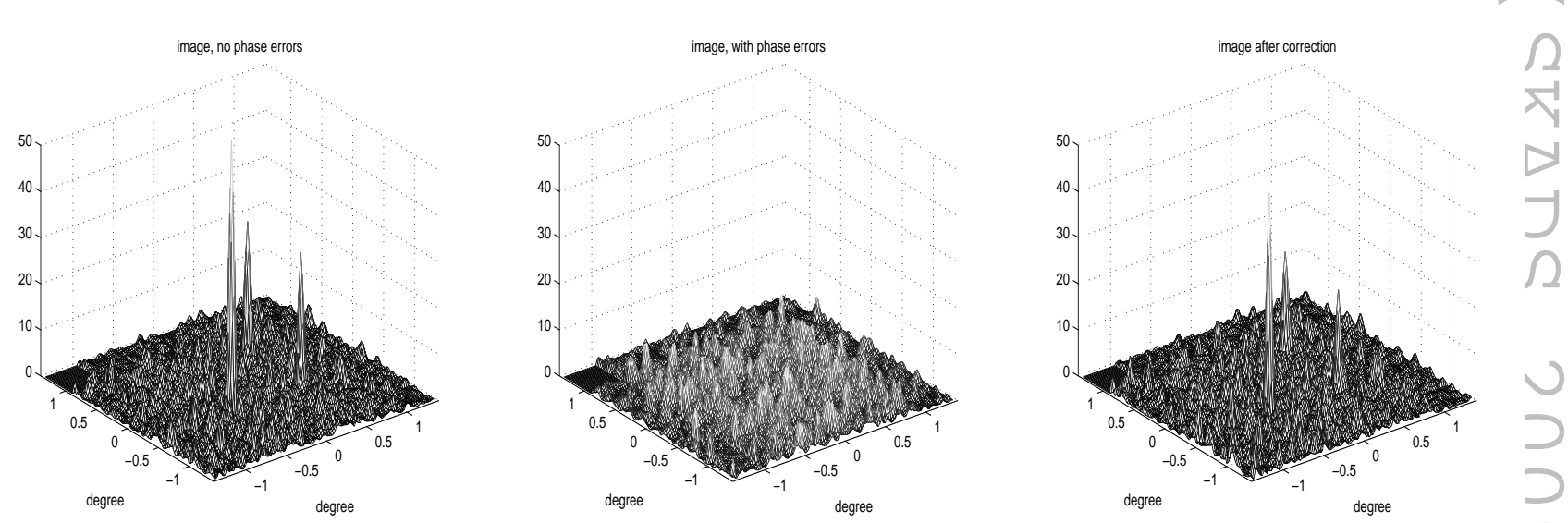

Fig. 5: a) left panel: synthesized image without phase errors; b) middle panel: synthesized image with phase errors; c) right panel: synthesized image after correction.
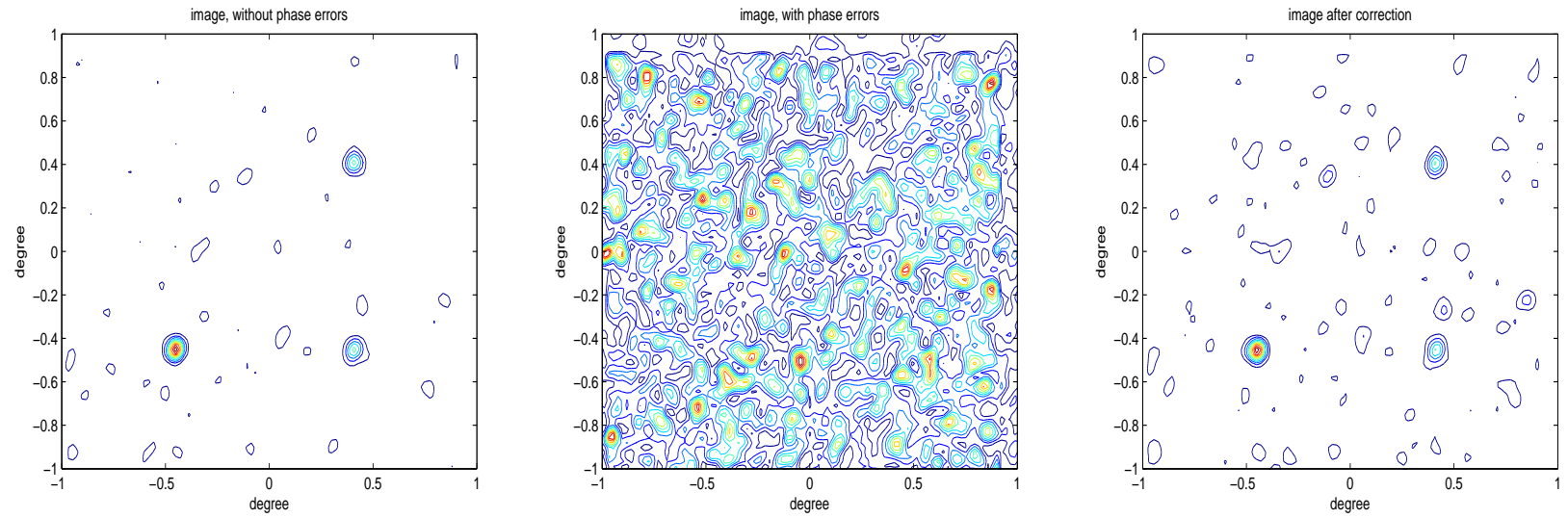

Fig. 6: Contour presentations, a) left panel: synthesized image without phase errors; b) middle panel: synthesized image with phase errors; c) right panel: synthesized image after correction. 